EPJ Web of Conferences 37, 01001 (2012)

DOI: $10.1051 /$ epjconf/20123701001

(C) Owned by the authors, published by EDP Sciences, 2012

\title{
Investigation of meson properties with the Belle detector
}

\author{
Simon Eidelman ${ }^{\mathrm{a}, 1,2}$ \\ 1 Budker Institute of Nuclear Physics SB RAS, Novosibirsk 630090, Russia \\ 2 Novosibirsk State University, Novosibirsk 630090, Russia
}

\begin{abstract}
We report on recent results from the Belle detector related to studies of meson properties. They include an observation of new mesons of light quarks in $\gamma \gamma$ collisions, a discovery of a new $D$ state in the $\psi$ family and observations of new states in the bottomonium family.
\end{abstract}

\section{Introduction}

Although $B$ factories were designed to study $\mathrm{CP}$ violation in the $B \bar{B}$ system at $\Upsilon(4 S)$ energy, since already ARGUS and CLEO times it has been known that much richer physics in this and other energy domains was accessible in specific initial states and /or with special methods of analysis, e.g., in $\gamma \rightarrow$ light quark mesons, decays of $\tau$ leptons and charm particles, production and decay of narrow $\Upsilon$ etc.

Huge statistics collected by BaBar $\left(\sim 550 \mathrm{fb}^{-1}\right)$ and Belle $\left(\sim 1030 \mathrm{fb}^{-1}\right)$ strengthened this understanding and resulted in principally new studies, e.g., $\gamma \gamma \rightarrow c \bar{c}$, initial-state radiation to $q \bar{q}$ and $c \bar{c}$. The combination of various methods and ideas led to spectacular observations in charmonium and bottomonium systems with many new states found as well as to detailed studies of various mesons of light quarks.

Progress of experiment stimulated theory resulting in many models: tetraquark, hybrid, molecules, hadrocharmonium or, alternatively, effects of close thresholds, coupled channels and rescattering.

Here we report on some selected results in the field of meson studies recently obtained at Belle.

\section{New light mesons between 2 and $3 \mathrm{GeV}$}

Belle used a data sample of $870 \mathrm{fb}^{-1}$ taken at $\Upsilon(n S), n=1, \ldots, 5$ to measure cross sections of $\gamma \gamma \rightarrow$ $\omega \phi, \phi \phi, \omega \omega[1]$. The invariant mass combinations shown in Fig. 1 show obvious structures below $3 \mathrm{GeV}$ in addition to charmonium signals.

2D angular analysis for various $J^{P}\left(0^{+}, 0^{-}, 2^{+}, 2^{-}\right)$reveals a mixture of spin- 0 and spin-2 components for all modes. Table 1 lists the positions (masses) of the new states as well as the corresponding cross sections at the peak.

In the charmonium energy range $\Gamma_{\gamma \gamma} \mathcal{B}(R \rightarrow V V)$ are measured with improved precision for the $\eta_{c}, \chi_{c 0}, \chi_{c 2} \rightarrow \phi \phi, \eta_{c} \rightarrow \omega \omega$ and upper limits for other decays to $\omega \omega, \omega \phi$ are the first measurements.

4-quark, t-channel factorization, one-pion exchange models fail to explain the position and height of the peaks.

\section{First $D$ state in charmonium family}

Using a full data sample of $772 \cdot 10^{6} B \bar{B}$ pairs at $\Upsilon(4 S)$ Belle studies $B^{+} \rightarrow \chi_{c 1} \gamma K^{+}$scanning a broad mass range. We identify $\chi_{c 1}$ by their decay to $J / \psi \gamma$ and $J / \psi$ by their decay into lepton pairs. In Fig. 2

\footnotetext{
a e-mail: S.I.Eidelman@inp.nsk.su
}

This is an Open Access article distributed under the terms of the Creative Commons Attribution License 2.0, which permits unrestricted use, distribution, and reproduction in any medium, provided the original work is properly cited. 
we show the invariant mass of the $\chi_{c 1} \gamma$ system. In addition to the obvious signal from the decay chain $B^{+} \rightarrow \psi(2 S) K^{+}, \psi(2 S) \rightarrow \chi_{c 1} \gamma$, a new state can be seen at $3823.5 \pm 2.8 \mathrm{MeV}$ with $4.2 \sigma$ significance. There is no signal at $3872 \mathrm{MeV}$.

The product of the branching fractions for the $X(3820)$ is measured to be $\mathcal{B}\left(B^{+} \rightarrow X(3820) K^{+}\right) \mathcal{B}\left(X \rightarrow \chi_{c 1} \gamma\right)=\left(9.7_{-2.5-1.0}^{+2.8+1.1}\right) \cdot 10^{-4}$.

It could be a ${ }^{3} D_{2}$ or $\psi(1 D)$ state expected at $3810-3840 \mathrm{MeV}$.

For $X(3872)$ a similar product of the branching fractions $\mathcal{B B}<1.9 \cdot 10^{-4} \Rightarrow \Gamma\left(X(3872) \rightarrow \chi_{c 1} \gamma\right) / \Gamma\left(X(3872) \rightarrow J / \psi \pi^{+} \pi^{-}\right)<0.26$ setting a constraint on the C-odd partner of $X(3872)$.

\section{New states in bottomonium}

Belle used $121.4 \mathrm{fb}^{-1}$ collected near $10860 \mathrm{MeV}$ to study $\Upsilon(5 S) \rightarrow X \pi^{+} \pi^{-}$, where $X=\Upsilon(1 S, 2 S, 3 S)$ or a really new $b \bar{b}$ state, using missing mass to $\pi^{+} \pi^{-}[2]$.

\section{$4.1 h_{b}(1 P)$ and $h_{b}(2 P)$ states}

Figure 3 shows the distribution of the missing mass to $\pi^{+} \pi^{-}$. A variety of states with different quantum numbers $J^{P}$ can be seen.

In addition to $\Upsilon(1 S, 2 S, 3 S)$, we observe $3 S \rightarrow 1 S$ and $2 S \rightarrow 1 S$ transitions, find weak evidence for the $\Upsilon(1 D)$ state with $2.4 \sigma$ significance and discover $h_{b}(1 P)$ and $h_{b}(2 P)$, for the latter - the first observation. In Table 2 we list the properties of the $h_{b}(n P)$ states.

It is also interesting to determine the value of the hyperfine splitting $\Delta M_{\mathrm{HF}}=<M\left(n^{3} P_{J}\right)>$ $-M\left(n^{1} P_{1}\right)$, where $\left\langle M\left(n^{3} P_{J}\right)>\right.$ is the spin-weighted average mass of the P-wave triplet states. Here a triplet $n^{3} P_{J}-\chi_{b J}(n P)$ and a singlet $n^{1} P_{1}-h_{b}(n P)$.

Thus, the hyperfine splitting is consistent with zero that can be compared to $0.00 \pm 0.15 \mathrm{MeV}$ for the $h_{c}(1 P)$. Another interesting observation following from a rather large cross section is that a spin flip of the $b$ quark is not suppressed as could be expected.

\subsection{Observation of Charged $Z_{b}(10610)$ and $Z_{b}(10650)$}

Analysis of $\Upsilon(5 S)$ decays to $h_{b}(1 P) \pi^{+} \pi^{-}, h_{b}(2 P) \pi^{+} \pi^{-}$as well as $\Upsilon(1 S) \pi^{+} \pi^{-}, \Upsilon(2 S) \pi^{+} \pi^{-}, \Upsilon(3 S) \pi^{+} \pi^{-}$ shows a resonant structure in $\Upsilon(n S) \pi, h_{b}(m P) \pi-Z_{b}$.

There are two $Z_{b}$ states at $10610 \mathrm{MeV}$ and $10650 \mathrm{MeV}$, which both decay into $\Upsilon(n S) \pi^{ \pm}$and $h_{b}(m P) \pi^{ \pm}, n=1,2,3 ; m=1$, 2, i.e., we observe $\Upsilon(5 S) \rightarrow Z_{b} \pi, Z_{b} \rightarrow \Upsilon(n S) \pi$ or $Z_{b} \rightarrow h_{b}(m P) \pi$. Two $Z_{b}$ states are charged and obviously exotic.

For each $Z_{b}$ state masses, widths, relative amplitudes measured in different decay modes are consistent. Relative phases are swapped for the $\Upsilon$ and $h_{b}$ final states as expected in the molecular model. Averaging the results from different decay modes one obtains the values of mass and width shown in Table 4. Results from five decay modes are also shown in Fig. 4.

\section{$4.3 \eta_{b}(1 S)$ and $\eta_{b}(2 S)$ states}

First claim for the observation of the $\eta_{b}(1 S)$ came in 2002 from ALEPH that reported an excess of six- and eight-prong events in $200 \mathrm{GeV} e^{+} e^{-}$collisions [4]. A position of the excess corresponded to a mass of $9300 \pm 20 \pm 20 \mathrm{MeV}$. However, as follows from the current measurements, most probably this was a fluctuation since its mass is significantly different from the recent values of BaBar $[5,6]$ and CLEO [7] that studied the $\Upsilon(2 S, 3 S) \rightarrow \eta_{b}(1 S) \gamma$ decays.

The world-average mass $M\left(\eta_{b}(1 S)\right)=9390.9 \pm 2.8 \mathrm{MeV}$ [8] corresponds to the hyperfine mass splitting $\Delta M_{\mathrm{hf}}=M(\Upsilon(1 S))-M\left(\eta_{b}(1 S)\right)=69.3 \pm 2.8 \mathrm{MeV}$, compared to $41 \pm 14 \mathrm{MeV}$ in pNRQCD [9] and $60 \pm 8 \mathrm{MeV}$ on the lattice [10]. No measurements of its width exist. 
It is tempting to search for $h_{b}(n P) \rightarrow \eta_{b}(m S) \gamma$ with $50 \mathrm{k}$ of $h_{b}(1 P)$ and $84 \mathrm{k}$ of $h_{b}(2 P)$ at Belle for which theory predicts sizable branchings [11]. Belle did that first with $121.4 \mathrm{fb}^{-1}$ and observed the $\eta_{b}(1 S)$ [12], then the analysis of the full data sample of $133.4 \mathrm{fb}^{-1}$ gave first evidence for the $\eta_{b}(2 S)[13]$.

The idea of the analysis is basically rather simple. We study the decay chain $\Upsilon(5 S) \rightarrow Z_{b}^{+} \pi^{-}$, followed by $Z_{b}^{+} \rightarrow h_{b}(n P) \pi^{+}$and then $h_{b}(n P) \rightarrow \eta_{b}(m S) \gamma$. We reconstruct only $\pi^{-}, \pi^{+}, \gamma$ and use corresponding missing masses to identify a signal. The missing mass to $\pi^{-}$is $M\left(Z_{b}^{+}\right)$, the missing mass to $\pi^{+} \pi^{-}$is $M\left(h_{b}\right)$, and that to $\pi^{+} \pi^{-} \gamma$ is $M\left(\eta_{b}\right)$. We define a variable $\Delta M_{\text {miss }}\left(\pi^{+} \pi^{-} \gamma\right) \equiv M_{\text {miss }}\left(\pi^{+} \pi^{-} \gamma\right)-$ $M_{\text {miss }}\left(\pi^{+} \pi^{-}\right)+M\left(h_{b}\right)$ and fit $M_{\text {miss }}\left(\pi^{+} \pi^{-}\right)$spectra in $\Delta M_{\text {miss }}\left(\pi^{+} \pi^{-} \gamma\right)$ bins. Figure 5 illustrates the method in the two-dimensional plot of $M_{\text {miss }}\left(\pi^{+} \pi^{-} \gamma\right)$ vs. $M_{\text {miss }}\left(\pi^{+} \pi^{-}\right)$.

In the ideal case all events group in the center, in reality there is finite resolution as well as background/fake $\pi$ and $\gamma$. The horizontal band for $\Delta M_{\text {miss }}\left(\pi^{+} \pi^{-} \gamma\right)$ corresponds to the $\eta_{b}$, true $\gamma$ and background $\pi^{+} \pi^{-}$. The vertical band for $M_{\text {miss }}\left(\pi^{+} \pi^{-}\right)$corresponds to $h_{b}$, true $\pi^{+} \pi^{-}$and background $\gamma$.

Using $133.4 \mathrm{fb}^{-1}$ and this method, Belle updated results on the $\eta_{b}(1 S)$ and reported first evidence for the $\eta_{b}(2 S)$, We have also updated the $h_{b}(1 P)$ and $h_{b}(2 P)$ mass measurements.

A simultaneous fit of $h_{b}(1 P) \rightarrow \eta_{b}(1 S)$ and $h_{b}(2 P) \rightarrow \eta_{b}(1 S)$ gives the following yields of the radiative transitions: for $h_{b}(1 P) \rightarrow \eta_{b}(1 S) \gamma-(23.5 \pm 2.0) \cdot 10^{3}$ events, for $h_{b}(2 P) \rightarrow \eta_{b}(1 S) \gamma-$ $(10.3 \pm 1.3) \cdot 10^{3}$ events. Finally, for $h_{b}(2 P) \rightarrow \eta_{b}(2 S) \gamma$ the yield is $(25.8 \pm 4.9) \cdot 10^{3}$ events.

In Tables 5 and 6 we summarize our results on the parameters of the $\eta_{b}(1,2 S)$ and $h_{b}(1,2 P)$ states as well as on the branching fractions of the $h_{b}$ radiative transitions to $\eta_{b}$ obtained from the full data sample at the $\Upsilon(10860)$.

\section{Conclusions}

- Huge data samples collected at $B$ factories together with various methods of analysis give access to rare processes in $e^{+} e^{-}$annihilation, $\gamma \gamma, B$ and $\Upsilon(5 S)$ decays.

- Many new mesons of light and heavy quarks were discovered, some expected and many with surprising or even exotic properties.

- Impressive progress is observed in the charmonium family studies, where about 20 new meson states were observed, but only a few fully understood and identified.

- In many cases detailed analysis of charmonium-like states is limited by statistics, a breakthrough is expected at $\operatorname{Super} B$-factories, PANDA and LHC.

- Various new states were discovered recently in the bottomonium family: $\eta_{b}(1 S), \eta_{b}(2 S), h_{b}(1 P)$, $h_{b}(2 P), Z_{b}(10610), Z_{b}(10650)$.

- Theoretical interpretation is very far from final and new interesting experimental observations are coming.

\section{Acknowledgments}

We greatly appreciate the efforts of the staff of KEKB to provide excellent operation of the complex. The author is grateful to the Conference organizers for a very interesting conference and kind support that made his attendance possible.

This work was partially supported by the Ministry of Education and Science of the Russian Federation, RFBR grants 10-02-00695, 11-02-00112, 11-02-00558, 12-02-00023, 12-02-01296, 12-02-91341 as well as DFG, GZ: HA 1457/7-2.

\section{References}

1. Z.-Q. Liu et al. (Belle Collab.), Phys. Rev. Lett. 108, (2012) 232001.

2. I. Adachi et al. (Belle Collab.), Phys. Rev. Lett. 108, (2012) 032001.

3. A. Bondar et al. (Belle Collab.), Phys. Rev. Lett. 108, (2012) 122001. 


\section{EPJ Web of Conferences}

Table 1. Positions of the new states and peak cross sections of their production

\begin{tabular}{|l|c|c|c|}
\hline Mode & $\omega \phi$ & $\phi \phi$ & $\omega \omega$ \\
\hline $\mathrm{M}, \mathrm{GeV}$ & 2.2 & 2.35 & 1.91 \\
\hline$\sigma_{\text {peak }}, \mathrm{nb}$ & $0.27 \pm 0.05$ & $0.30 \pm 0.04$ & $5.30 \pm 0.42$ \\
\hline
\end{tabular}

Table 2. Properties of the $h_{b}$ states

\begin{tabular}{|l|r|c|r|}
\hline State & Yield, 10 & Mass, MeV & Sign. \\
\hline$h_{b}(1 P)$ & $50.4 \pm 7.8_{-9.1}^{+4.5}$ & $9898.3 \pm 1.1_{-1.1}^{+1.6}$ & $5.5 \sigma$ \\
\hline$h_{b}(2 P)$ & $84.4 \pm 6.8_{-10 .}^{+23 .}$ & $10259.8 \pm 0.6_{-1.0}^{+1.4}$ & $11.2 \sigma$ \\
\hline
\end{tabular}

Table 3. Hyperfine splitting and cross sections of $h_{b}(n P)$ production

\begin{tabular}{|l|c|c|}
\hline State & $h_{b}(1 P)$ & $h_{b}(2 P)$ \\
\hline$\Delta M_{\mathrm{HF}}, \mathrm{MeV}$ & $1.6 \pm 1.5$ & $+0.5_{-1.2}^{+1.6}$ \\
\hline$\frac{\sigma\left(h_{b}(n P) \pi^{+} \pi^{-}\right)}{\sigma\left(\Upsilon(2 S) \pi^{+} \pi^{-}\right)}$ & $0.46 \pm 0.08_{-0.12}^{+0.07}$ & $0.77 \pm 0.08_{-0.17}^{+0.22}$ \\
\hline
\end{tabular}

Table 4. Mass and width of the $Z_{b}$ states

\begin{tabular}{|l|c|c|}
\hline State & $Z_{b}(10610)$ & $Z_{b}(10650)$ \\
\hline$M, \mathrm{MeV}$ & $10607.2 \pm 2.0$ & $10652.2 \pm 1.5$ \\
\hline$\Gamma, \mathrm{MeV}$ & $18.4 \pm 2.4$ & $11.5 \pm 2.2$ \\
\hline
\end{tabular}

Table 5. Results on $\eta_{b}$ and $h_{b}$ states from the full data sample

\begin{tabular}{|l|c|c|c|}
\hline State & Mass, MeV & Width, MeV & $\Delta M_{\mathrm{hf}}, \mathrm{MeV}$ \\
\hline$\eta_{b}(1 S)$ & $9402.4 \pm 1.5 \pm 1.8$ & $10.8_{-3.7-2.0}^{+4.0+4.5}$ & $57.9 \pm 2.3$ \\
\hline$\eta_{b}(2 S)$ & $9999.0 \pm 3.5_{-1.9}^{+2.8}$ & $<24$ & $24.3_{-4.5}^{+4.0}$ \\
\hline$h_{b}(1 P)$ & $9899.1 \pm 0.4 \pm 1.0$ & - & $0.8 \pm 1.1$ \\
\hline$h_{b}(2 P)$ & $10259.8 \pm 0.5 \pm 1.1$ & - & $0.5 \pm 1.2$ \\
\hline
\end{tabular}

Table 6. Branching fractions of $h_{b}(n P) \rightarrow \eta_{b}(m S)$ transitions

\begin{tabular}{|c|c|c|c|}
\hline $\mathcal{B}, \%$ & $1 P \rightarrow 1 S$ & $2 P \rightarrow 1 S$ & $2 P \rightarrow 2 S$ \\
\hline- & $49.2 \pm 5.7_{-3.3}^{+5.6}$ & $22.3 \pm 3.8_{-3.3}^{+3.1}$ & $47.5 \pm 10.5_{-7.7}^{+6.8}$ \\
\hline
\end{tabular}
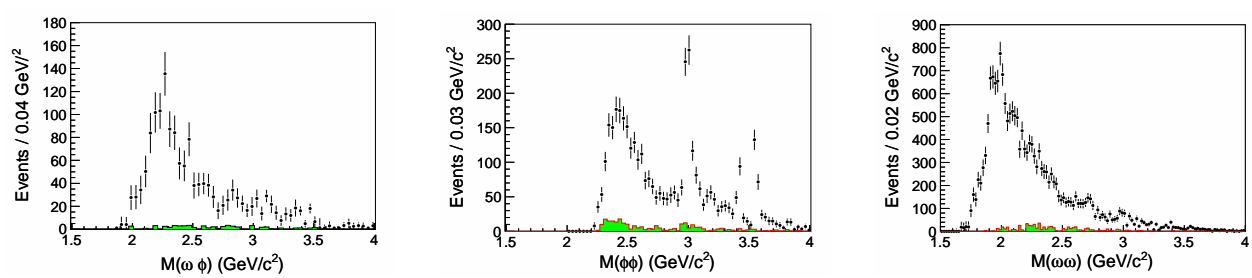

Fig. 1. The invariant mass distributions for (a) $\omega \phi$, (b) $\phi \phi$ and (c) $\omega \omega$. The shaded histograms are from the corresponding normalized sidebands. 


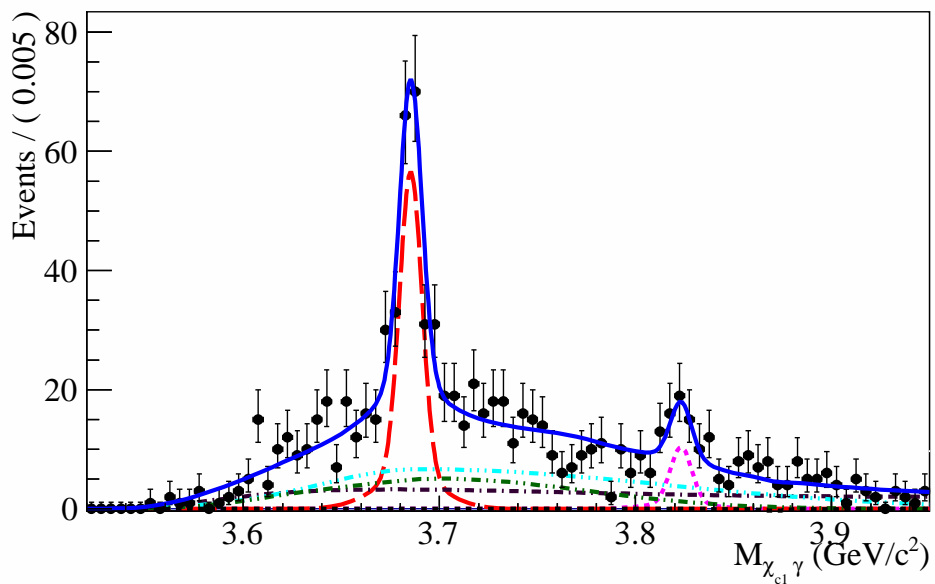

Fig. 2. The invariant mass of the $\chi_{c 1} \gamma$ system

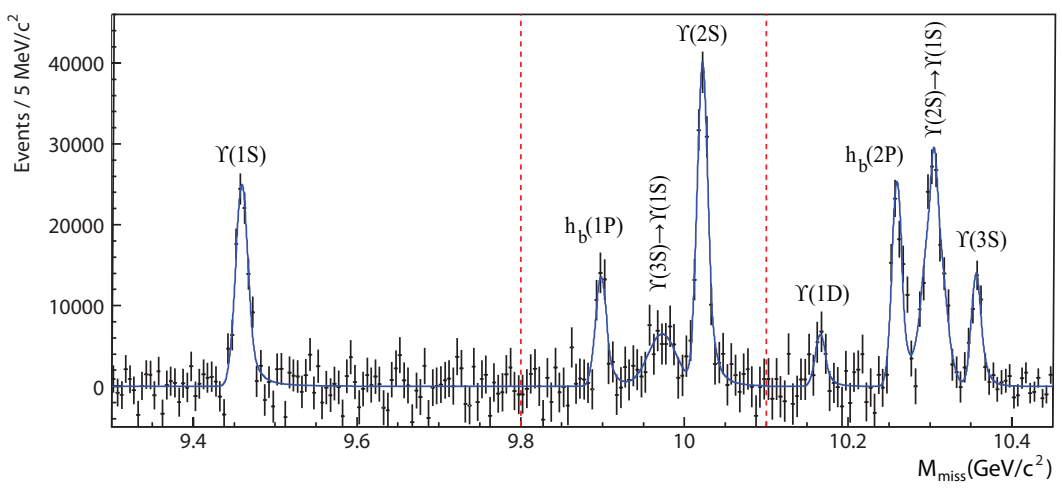

Fig. 3. Distribution of the missing mass to $\pi^{+} \pi^{-}$

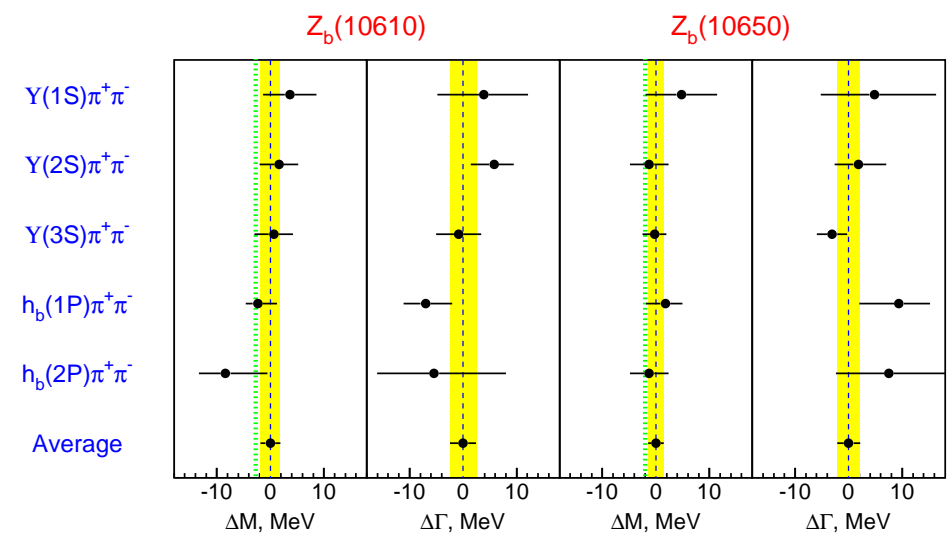

Fig. 4. Comparison of mass and width of the $Z_{b}$ states in 5 decay modes 

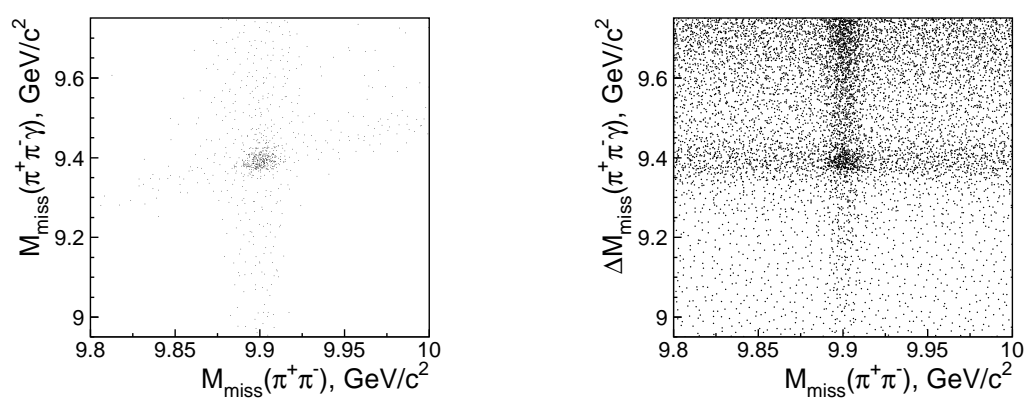

Fig. 5. Search for $\eta_{b}$ states. Left - Monte Carlo, right - data

4. A. Heister et al. (ALEPH Collab.), Phys. Lett. B 530, (2002) 56.

5. B. Aubert et al. (BaBar Collab.), Phys. Rev. Lett. 101, (2008) 071801.

6. B. Aubert et al. (BaBar Collab.), Phys. Rev. Lett. 103, (2009) 161801.

7. G. Bonvicini et al. (CLEO Collab.), Phys. Rev. D 81, (2010) 031104.

8. K. Nakamura et al. (Particle Data Group), J. Phys. G 37, (2010) 075021.

9. B.A. Kniehl et al., Phys. Rev. Lett. 92, (2004) 242001.

10. S. Meinel, Phys. Rev. D 82, (2010) 114502.

11. S. Godfrey and J.L. Rosner, Phys. Rev. D 66, (2002) 014012.

12. I. Adachi et al. (Belle Collab.), arxiv:1110.3934.

13. R. Mizuk et al. (Belle Collab.), arxiv:1205.6351. 